\title{
Desafios e perspectivas do cuidado em enfermagem a populações em situação de vulnerabilidade
}

\author{
Challenges and perspectives of nursing care to vulnerable populations \\ Desafíos y perspectivas del cuidado en Enfermería a poblaciones en situación \\ de vulnerabilidad

\section{Rosana Maffacciollia Dora Lúcia Leidens Corrêa de Oliveira ${ }^{b}$}

Como citar este artigo:

Maffacciolli R, Oliveira DLLC. Desafios e perspectivas do cuidado em enfermagem a populações em situação de vulnerabilidade. Rev Gaúcha Enferm. 2018;39:e20170189.doi: https://doi. org/10.1590/1983-1447.2018.20170189. aniversidade Federal do Rio Grande do Sul (UFRGS), Escola de Enfermagem, Programa de Pós- Graduação em Enfermagem. Porto Alegre, Rio Grande do Sul, Brasil.

- Universidade Federal do Rio Grande do Sul (UFRGS), Escola de Enfermagem. Porto Alegre, Rio Grande do Sul, Brasil.

\section{RESUMO}

Objetivo: Refletir sobre o cuidado em Enfermagem a populações em situação de vulnerabilidade. Método: Ensaio teórico-reflexivo, embasado no referencial da Vulnerabilidade e Direitos Humanos.

Resultados: A abordagem psicossocial da vulnerabilidade permitiu ressignificar a noção de necessidades de saúde de populações em situação de vulnerabilidade a partir de um posicionamento favorável ao enfrentamento das violações de direitos que marcam o viver dessas pessoas. Também permitiu problematizar a utilização da expressão "populações-chave" para priorizar esses grupos em ações de saúde e seus desdobramentos normativos e moralizantes contrários à proteção de direitos e aos fundamentos humanistas do cuidado. Conclusão: Ao desenvolver esses argumentos, concluiu-se que, para superar os desafios do cuidado em Enfermagem a essas populações, é imprescindível assimilar novas perspectivas sobre a existência humana a partir da defesa de sua diversidade e da igualdade de direitos.

Palavras-chave: Cuidados de enfermagem. Vulnerabilidade em saúde. Direitos humanos.

\section{ABSTRACT}

Objective: Analyze perspectives of nursing care provided to vulnerable populations.

Method: This is a theoretical-reflective study based on the Vulnerability and Human Rights framework.

Results: The psychosocial approach to vulnerability has allowed the resignification of the notion of the health needs of vulnerable populations through a position that favors addressing right violations that mark the life of these people. It has also allowed to develop a discussion on the use of the expression 'key populations' to prioritize these groups in health actions and their normative and moralizing developments contrary to right protection and the humanistic foundations of care.

Conclusion: When developing these arguments, this study concluded that, in order to overcome the challenges of providing nursing care to these populations, new perspectives about the human existence should be considered starting from the defense of their diversity and equal rights.

Keywords: Nursing care. Health vulnerability. Human rights.

\section{RESUMEN}

Objetivo: Reflexionar sobre el cuidado en Enfermería a poblaciones en situación de vulnerabilidade.

Método: Ensayo teórico-reflexivo, basado en el referencial de la Vulnerabilidad y Derechos Humanos.

Resultados: El abordaje psicosocial de la vulnerabilidad permitió resignificar la noción de necesidades de salud de poblaciones en situación de vulnerabilidad a partir de un posicionamiento favorable al enfrentamiento de las violaciones de derechos que marcan el vivir de esas personas. También permitió problematizar la utilización de la expresión "poblaciones clave" para priorizar esos grupos en acciones de salud y sus desdoblamientos normativos y moralizantes contrarios a la protección de derechos ya los fundamentos humanistas del cuidado.

Conclusión: Al desarrollar estos argumentos, se concluyó que, para superar los desafíos del cuidado en Enfermería a esas poblaciones, es imprescindible asimilar nuevas perspectiva sobre la existencia humana a partir de la defensa de su diversidade y de la igualdad de derechos.

Palabras clave: Atención de enfermería. Vulnerabilidad en salud. Derechos humanos. 


\section{—INTRODUÇÃO}

A implementação de práticas de cuidado que respondam às necessidades de populações em situação de vulnerabilidade constitui um dos principais desafios para o campo da saúde. No sentido de apoiar a inovação dessas práticas, observa-se intenso interesse, especialmente nas últimas três décadas, em aprofundar o conhecimento sobre desigualdades sociais, sua relação com a precarização das condições de vida e a constituição de vulnerabilidades em saúde. Nesse contexto, a inovação analítica posta pelo conceito de vulnerabilidade, surge para ampliar o entendimento sobre as situações que determinam os piores desfechos em saúde em cenários de iniquidades sociais (desigualdades resultantes de injustiças e exclusão sociall)(-2).

Na história recente, a complexidade desses cenários e suas consequências para a saúde teve sua maior expressão durante a pandemia da aids. Nos anos 1990, a doença avançava nas populações com baixo poder aquisitivo, com pouco acesso a bens e serviços essenciais para a manutenção à saúde e inferiorizadas pelo seu pertencimento de gênero, de raça e pelo exercício de sua sexualidade. A vulnerabilidade, desde então, figura como categoria conceitual que consegue abarcar os efeitos de um cenário cultural e político reprodutor de injustiças e de violação de direitos humanos(1).

O conceito de vulnerabilidade atrelada ao conhecimento sobre direitos humanos mudou a resposta programática à aids, especialmente no Brasil onde, desde a década de 1990, o Sistema Único de Saúde (SUS), regido pelos princípios da universalidade, integralidade e equidade, oferta atenção em saúde voltada a amenizar os efeitos deletérios dessas iniquidades sociais ${ }^{(1)}$.

Com uma expressiva força de trabalho no SUS, a Enfermagem tem condições de colaborar para a consolidação dessas transformações. É necessário, porém, aprofundar o conhecimento sobre vulnerabilidades em saúde, no sentido de entender os desafios que os contextos marcados por iniquidades sociais impõem a atuação desses profissionais. Diversas publicações nacionais e internacionais vêm difundindo esse conhecimento. No âmbito brasileiro, algumas publicações problematizam as explicações binárias sobre as causas dos problemas, a pouca valorização da subjetividade das pessoas e as dificuldades de relacionamento interpessoal com grupos considerados moralmente desviantes. Publicações internacionais tematizam essa questão observando a importância de se considerar a posição social e a inserção cultural das pessoas sob cuidados, de adotar uma postura profissional empática e promotora da justiça social e ofertar cuidado a partir de uma perspectiva psicossocial das condições dessas populações(3-8).

Apoiado no referencial da vulnerabilidade e direitos humanos, o artigo procurou contribuir com um debate que ainda é incipiente na Enfermagem. O objetivo foi refletir sobre o cuidado em Enfermagem a populações em situação de vulnerabilidade, examinado o contexto de violação de direitos humanos que marca o viver dessas pessoas.

\section{Desafios do cuidado em enfermagem às populações em situação de vulnerabilidade}

Mantendo-se como referência para a qualificação das habilidades técnicas e dos saberes científicos, o cuidado é conceito estruturante para a Enfermagem, sendo destacados entre seus fundamentos humanistas, a empatia, colaboração e solidariedade ${ }^{(9)}$. Ao se guiar pelo agir solidário, a Enfermagem respeita as razões socioculturais de cada cidadão, assimilando experiências dolorosas e alegres que somente a convivência proporciona, o que resulta em simetria e equilíbrio nas suas múltiplas atividades ${ }^{(10)}$.

Embora o ato de cuidar e suas prerrogativas humanistas já ancore as atividades exercidas pelos profissionais de enfermagem desde tempos remotos, no âmbito da saúde brasileira, esse tema é foco de debate. A implementação, em 2003, da Política Nacional de Humanização (PNH), representa um dos desdobramentos desse debate ao concretizar um movimento em prol da garantia de direitos de usuários e trabalhadores que ambicionava reinventar a clínica, a gestão e modos tradicionais do trabalho em saúde(11).

No Brasil, a representativa inserção dos profissionais de enfermagem no setor saúde, remete ao reconhecimento da sua importância para a qualidade do cuidado. Neste sentido, e considerando a premência em atender as necessidades de saúde das populações em situação de vulnerabilidade, duas questões se impõem. Quais desafios precisam ser enfrentados para efetivar o cuidado a essas populações? De que modo esses desafios nos aproximam ou nos distanciam da prática de um cuidado humanizado?

Para apontar o caminho a ser trilhado na busca por responder a tais questões é importante compreendermos que a gama de situações e condições individuais e sociais, requeridas nas análises da vulnerabilidade, é demandante de um exercício analítico que permita desvelar vulnerabilidades concretas na vida cotidiana das pessoas. A perspectiva que vem evidenciando o papel das violações de direitos humanos é profícua em responder a essa demanda, pois sugere que trajetórias pessoais e psicossociais dependem de relações de poder que só podem ser compreendidas em termos de seus significados locais e contexto estrutu- 
rais. Para essa perspectiva, contextos em que relações de poder estruturam desigualdades de classe social, de raça, de gênero e sexualidade definem trajetórias pessoais de vulnerabilidade ${ }^{(1)}$. Essa concepção nos leva a questionar a abrangência de sentidos vinculados à terminologia "populações vulneráveis", sobretudo sobre seu uso para designar um grupo genérico de pessoas afetadas por problemas semelhantes no âmbito da sua saúde e da sua condição socioeconômica. Nossas experiências em pesquisas sobre tuberculose têm nos estimulado a formular essa crítica, ao evidenciarmos que os piores desfechos do adoecimento guardam relação com estereótipos negativos associados à pobreza, à Aids e ao uso de drogas ilícitas; com o rechaço moral na vivência velada do racismo e da homofobia; e com a submissão e opressão de mulheres que as expunha à infecção pelo HIV(12).

Essas experiências também têm nos oportunizado aprimorar o conhecimento sobre as necessidades implicadas nos processos de adoecimento dessas pessoas, o que certamente deveria impactar os planos de cuidado elaborados para cada uma delas. Nesse sentido, mais importante que enumerar e classificar necessidades (como moradia, vínculos sociais, renda, segurança e alimentação), é compreender seus significados e implicações na vida cotidiana. Trata-se de um exercício reflexivo que não encontra as condições para seu êxito quando a dinâmica relacional, que aproxima profissionais de saúde e usuários, desconsidera uma escuta atenta, sensível e potente em produzir respostas compartilhadas e condizentes com as interpelações do ambiente em que a vida acontece ${ }^{(2)}$. Na linha do que estamos apontando a respeito das populações em situação de vulnerabilidade, assumir uma postura coerente com essa dinâmica relacional requer que enfrentemos um paradoxo. Trata-se da consciência do cuidado humanizado como pressuposto para a qualidade das práticas em saúde em confronto com as discrepâncias identitárias entre os interlocutores - profissionais e usuários - que protagonizam a ação do cuidado.

As diferenças sociais que aqui ressaltamos se referem às marcas simbólicas e materiais encarnadas nas pessoas que vivem cotidianamente situações de vulnerabilidade em relação a pessoas com experiências identitárias diametralmente diversas. Vale destacar que diferença $a^{(13)}$, como categoria sociológica, nos ajuda esclarecer que a diferenciação social é um processo categorizador das pessoas em função de pertencerem a certos grupos ou de aderirem a certas práticas, podendo aprofundar desigualdades sociais, a depender do modo como esses discursos operam em cada contexto analisado. Esse debate perpassa o exame das relações entre diferentes para que possamos refletir sobre o quanto é desafiador promover um agir solidário e uma prática cuidadora nos termos da humanização, em meio a assimetrias e desequilíbrios nas relações sociais. Outro desafio que cabe agregar aqui refere-se a julgar se, nas relações que estabelecemos com as pessoas em contextos de desigualdade social, envidamos esforços para desocultar opressão, violência, exploração e outras manifestações de violação de direitos humanos, encarando-as como fontes de sofrimento e adoecimento. No tópico a seguir, manteremos a exposição dessas ideias repousando-as nos pressupostos dos direitos humanos em sua interlocução com a saúde. Adotando essa via analítica, propomos iluminar o debate sobre as perspectivas de se produzir práticas de cuidado que impactem na melhoria da saúde das populações em situação de vulnerabilidade.

\section{A prática do cuidado em contextos de vulnerabilidades e violação de direitos humanos}

Não discriminação, igualdade e participação das comunidades em decisões sobre o enfrentamento aos problemas que as afetam são princípios dos direitos humanos centrais para responder a situações de vulnerabilidade. Tais princípios têm representado a inserção de inovações ao efetivar novas formas de lidar com necessidades de saúde nas agendas políticas nacionais, bem como na oferta de diretrizes programáticas em nível global(14).

No Brasil, as produções que incorporam esse referencial ainda são incipientes ${ }^{(1)}$, mas o interesse tende a se intensificar, especialmente em áreas como a Enfermagem, em vista dos desafios colocados para a prática de um cuidado humanizado e resolutivo.

Uma instigante fonte de conhecimentos tem sido as produções de pesquisadores brasileiros no quadro da Vulnerabilidade e Direitos Humanos, ao proporem novas tecnologias de prevenção e atenção em saúde com maior sensibilidade às necessidades, valores e relações intersubjetivas concretas entre os atores envolvidos nas cenas de cuidado. Um elemento constante nessas proposições é a intransigente renúncia aos universalismos e essencialismos de identidades muito abstratas ou estereotipadas, suscitados pela noção de "populações-alvo"(2). Essa noção é bastante utilizada para priorizar, nos programas de saúde, grupos afetados por semelhantes condições no que se refere a desvantagens sociais, e nesse sentido "populações vulneráveis", como aqui estamos problematizando, seria a terminologia com maior eficácia retórica em representá-los. 
Esse posicionamento, apoiado em análises psicossociais da vulnerabilidade, procura alertar para as distopias geradas em representações genéricas de pessoas, por exemplo, "não aderente ao tratamento", "usuário de drogas", "sem estudo". No âmbito da saúde, podemos identificar como consequências dessas distopias, as condutas que "moralizam ou normatizam a priori qualquer prática ou concepção do que seja bom", respaldadas em conhecimento científico altamente persuasivo em receitar comportamentos "saudáveis"(2). Essa crítica facilita a compreensão de processos históricos em que violações de direitos humanos têm papel fundamental nas trajetórias das pessoas, contrapondo-se à tendência ao julgamento moral dos seus comportamentos. Ainda, nos impulsiona a afastar a ideia de que, por pertencer a determinado grupo, o outro é naturalmente displicente, ameaçador, incapaz ou detentor de outros qualitativos que o desabilitam para a aceitação social plena. Os processos sócios históricos relacionados à deterioração da identidade de certos grupos foram extensamente estudados na sociologia(15), cabendo aqui referi-los como conhecimento central na compreensão das condutas discriminatórias adotadas em muitas manifestações da vida social, inclusive no contexto das relações institucionais no campo da saúde. Descobrir quem, de fato, é a pessoa com quem mantemos essas relações, sendo ela respeitada em sua incontestável dignidade como ser humano, amplia as chances de reconhecermos suas singularidades emocionais, intelectuais e culturais expressadas em seus cotidianos de vida e vinculantes dos sentidos produzidos em suas histórias. Assimilar a diversidade da existência humana é, portanto, parte de uma proposta de mudanças nos padrões de socialização que visam o cuidado em saúde.

Nas propostas de cuidado a populações em situação de vulnerabilidade, é fundamental compreender a diversidade humana para além das estereotipias de um cenário cultural marcado pela exclusão social. No campo da saúde, esse cenário ganha contornos ainda mais específicos com a reprodução de uma visão reducionista da realidade das pessoas, forjada pela necessidade de decompor essa realidade em objetos mais aptos a serem conhecidos e dominados pelos profissionais da saúde ${ }^{(16)}$.

Uma inspiradora narrativa sobre cuidado em saúde nos levou a reconhecer a importância de contemplar pressupostos humanistas nas ações de natureza técnica, de modo não reproduzir esses reducionismos. Nessa narrativa, a história de um "projeto de felicidade" interrompido é resgatada no momento dar resposta à desfavorável condição clínica de "dona Violeta", que ao perder a perspectiva de viver na casa projetada com seu companheiro, perde também as referências de sua existência. Não embasar a prática de cuidado em duros preceitos técnicos das ciências da saúde foi fundamental para aliviar as "dores de saudade" no seu peito(12).

Em cenários socioculturais de desrespeito aos direitos humanos, portanto, de reduzidas chances em concretizar "projetos de felicidade", poderíamos questionar quais condutas em saúde seriam eficazes em extinguir as dores que alcançam a alma de um corpo cotidianamente atingido pelo racismo e pela discriminação de classe social? Da mesma forma, como remediar essas dores em mulheres que precisam abrir mão da liberdade ao exercerem um papel que para elas é pré-determinado antes de nascerem? E para quem se aventura a ter experiências homossexuais ou adotar uma identidade de gênero destoante de normas heterossexuais e sexistas, quais seriam as dores e quais intervenções poderiam mitigá-las? O aparato da saúde seria hábil em reverter as sequelas da estigmatização em usuários de drogas e pessoas vivendo com HIV? Acreditamos que ao trilhar os caminhos que levam a responder a essas questões, a Enfermagem pode acumular novos conhecimentos que a instrumentaliza a conduzir práticas de cuidado balizadas em fundamentos humanistas em contextos de vulnerabilidade e violação de direitos humanos.

\section{— CONSIDERAÇÕES FINAIS}

As reflexões apresentadas nesse artigo permitiram concluir que os desafios ao cuidado em Enfermagem a populações em situação de vulnerabilidade, podem ser superados ao ampliarmos nossas perspectivas sobre a existência humana a partir de um posicionamento ético compatível com a defesa de liberdades, diversidades e não discriminação. A escassez de produções na Enfermagem sobre abordagens que integram os direitos humanos e o cuidado em saúde pode ser fator limitante do alcance das reflexões apresentadas neste artigo, porém as apresentamos como um convite à inovação que a própria realidade demanda ao evidenciarmos contextos cada vez mais complexos de vulnerabilidade em saúde. Como uma das áreas profissionais que mais densamente ocupa espaços de atuação no setor da saúde no Brasil, acreditamos que a Enfermagem se beneficia desses conhecimentos, ao aperfeiçoar suas práticas e colabora significativamente para a concretização das propostas progressistas do SUS, especialmente aquelas que se referem ao direito a um cuidado integral e humanizado em saúde. Cabe, como recomendações, ampliar as oportunidades de analisar essas práticas a fim de consolidar o conhecimento sobre vulnerabilidade e direitos humanos na enfermagem. 


\section{REFERÊNCIAS}

1. Ayres JR, Paiva V, França Junior IF. Conceitos e práticas de prevenção: da história natural da doença ao quadro da Vulnerabilidade e Direitos Humanos. In: Paiva $V$, Ayres JR, Buchalla CM, organizadores. Vulnerabilidade e direitos humanos: prevenção e promoção da saúde: da doença à cidadania. Curitiba: Juruá; 2012. p. 71-94.

2. Paiva V. Cenas da vida cotidiana: metodologia para compreender e reduzir a vulnerabilidade na perspectiva dos direitos humanos. In: Paiva V, Ayres JR, Buchalla CM, organizadores. Vulnerabilidade e direitos humanos: prevenção e promoção da saúde: da doença à cidadania. Curitiba: Juruá; 2012. p. 165-208.

3. Silva IR, Gomes AMT, Valadares GV, Santos NLP, Silva TP, Leite JL. Nurses' perceptions of the vulnerabilities to STD/AIDS in light of the process of adolescence. Rev Gaúcha Enferm. 2015 Sep;36(3):72-8. doi: https://doi.org/10.1590/19831447.2015.03.47293.

4. Zanatta EA, Motta MGC. Violência, sob o olhar de jovens, na perspectiva da corporeidade e da vulnerabilidade. Texto Contexto Enferm. 2015 June;24(2):47685. doi: https://doi.org/10.1590/0104-07072015001302014.

5. Pedrosa SM, Reis ML, Gontijo DT, Teles SA, Medeiros M. The path to crack addiction: perceptions of people under treatment. Rev Bras Enferm. 2016;69(5):899906. doi: https://doi.org/10.1590/0034-7167-2016-0045.

6. Sinkfield-Morey T. Diversity, inclusion, and storying: connecting across cultures to give meaning to patients' whole health. Creat Nurs. 2018;24(1):12-9. doi: https://doi.org/10.1891/1078-4535.24.1.12.

7. Hellman AN, Cass C, Cathey H, Smith SL, Hurley S. Understanding poverty: teaching social justice in undergraduate nursing education. J Forensic Nurs. 2018;14(1):11-17. doi: https://doi.org/0.1097/JFN.0000000000000182.
8. McCann E. Vulnerability and psychosocial risk factors regarding people who identify as transgender: a systematic review of the research evidence. Issues Mental Health Nurs. 2018;39(1):3-15. doi: https://doi.org/10.1080/0161284 0.2017 .1382623

9. Waldow VR. Cuidar: expressão humanizadora da enfermagem. 2. ed. Petrópolis: Vozes; 2007.

10. Souza ML, Sartor VVB. [Nursing care: a theoretical approcah]. Texto Contexto Enferm. 2005;14(2):266-70. doi: https://doi.org/10.1590/5010407072005000200015. Portuguese.

11. Martins CP, Luzio CA. Política HumanizaSUS: ancorar um navio no espaço. Interface (Botucatu). 2017;21(60):13-22. doi: https://doi.org/10.1590/180757622015.0614.

12. Maffacciolli R, Oliveira DLLC, Brand EM. Vulnerabilidade e direitos humanos: compreensão de trajetórias de internação por tuberculose. Saude Soc 2017;26(1):286-99. doi: https://doi.org/10.1590/s0104-12902017168038.

13. Brah A. Difference, diversity, differentiation. Cadernos Pagu. 2006;(26):329-76. doi: https://doi.org/10.1590/S0104-83332006000100014.

14. Ferguson L, Tarantola D, Hoffmann M, Gruskin S. Non-communicable diseases and human rights: Global synergies, gaps and opportunities. Global Public Health. 2017;12(10):1200-27. doi: https://doi.org/10.1080/17441692.2016. 1158847.

15. Goffman E. Estigma: notas sobre a manipulação da identidade deteriorada. 4. ed. Rio de Janeiro: LTC; 2008.

16. Ayres JRCM. Revisitando "D. Violeta": nos caminhos do cuidado. In: Pinheiro R, Müller Neto JS, Spinelli, MAS, Silva Júnior AG, organizadores. Construção social da demanda por cuidado: revisitando o direito à saúde, 0 trabalho em equipe, os espaços públicos e a participação. Rio de Janeiro: UERJ/IMS/CEPESC/LAPPIS/ ABRASC0; 2013. p.15-30.
Autor correspondente:

Rosana Maffacciolli

E-mail: rosanamaffac@yahoo.com.br
Recebido: 15.09.2017

Aprovado: 18.05.2018 